\title{
Enhanced Lanthanide Luminescence Using Silver Nanostructures: Opportunities for a New Class of Probes with Exceptional Spectral Characteristics
}

\author{
Meng Wu, ${ }^{1}$ Joseph R. Lakowicz, ${ }^{1,3}$ and Chris D. Geddes ${ }^{1,2,3}$
}

Received July 10, 2004; accepted July 14, 2004

\begin{abstract}
The photophysical effects of europium tetracycline immobilized in thin polyvinyl alcohol films coated onto silver nanostructures have been investigated. Complimentary to recent reports from our laboratories that the close proximity of luorophores to silver nanostructures can enhance their intrinsic radiative decay rate, we show that up to a 16 -fold enhancement in lanthanide luminescence is possible, accompanied by a notable reduction in luminescence lifetime. These results suggest the potential future development of a new class of significantly brighter lanthanide based probes with exceptional spectral properties, which can probably undergo significantly more excitation-emission event cycles due to the reduced lifetime, substantially increasing detectability.
\end{abstract}

KEY WORDS: Europium; tetracycline; metal-enhanced fluorescence; radiative decay engineering; enhanced lanthanide luminescence; silver island films; luminescence; silver nanostructures.

\section{INTRODUCTION}

In recent reports from our laboratories we have described the favorable effects of nanosecond decay time fluorophores near-to silver nanoparticles: silver Island films ( $\mathrm{SiFs})$ [1-6]; silver colloids films [7]; solution based silver colloids [8]; silver nanorods [9]; and fractal-like silver surfaces [10-13]. These effects include increased quantum yields, decreased fluorophore lifetimes, increased photostability, and increased rates of energy transfer. These effects are due to the interactions of the excited-state fluorophores with the surface plasmon resonances on the surface of the metal [14-17]. These interactions can result in

\footnotetext{
${ }^{1}$ Center for Fluorescence Spectroscopy, Department of Biochemistry, and Molecular Biology, Medical Biotechnology Center, University of Maryland School of Medicine, 725 West Lombard Street, Baltimore, Maryland 21201.

${ }^{2}$ Institute of Fluorescence, Laboratory for Advanced Fluorescence Spectroscopy, Medical Biotechnology Center, University of Maryland Biotechnology Institute, 725 West Lombard Street, Baltimore, Maryland 21201.

${ }^{3}$ To whom correspondence should be addressed. E-mail: geddes@ umbi.umd.edu; lakowicz@cfs.umbi.umd.edu
}

increased rates of excitation, increased intensities (quantum yields), and even quenching [18]. For our applications of enhanced fluorescence/luminescence and therefore luminophore detectability, the most important effect is an increase in the radiative decay rate of fluorophores, $\Gamma$, which occurs for fluorophores positioned $\approx 50-200 \AA$ from the metallic surface $[14,17]$. We have subsequently referred to these interactions as both metal-enhanced fluorescence [17] and radiative decay engineering [14]. In nearly all examples of fluorescence, the fluorophores or luminophores are examined in the free space condition, that is, the fluorophore can be described as an oscillating dipole radiating energy into a transparent and homogeneous medium. Although some fluorescent probes are known to be sensitive to the local environment, such as polarity, quenching, and energy transfer [19], these interactions modify the nonradiative decay pathways and not the free space spectral properties $[14,15,17]$.

ABBREVIATIONS: SiFs, Silver Island Films; EuTc, Europium (III) Tetracycline complex; PVA, Polyvinyl alcohol; S, Silvered surface; $\mathrm{G}$, Glass surface (control sample); $\tau$, Amplitude weighted lifetime; $\bar{\tau}$, Mean lifetime. 
In this report, we show how metallic particles can indeed modify the radiative decay rate of a Europium complex, producing favorable enhanced luminescence intensities, and reduced lifetimes, as compared to the free space condition, where it is not possible to notably alter the radiative decay rate. The lanthanides display decay times on the $\mu \mathrm{s} / \mathrm{ms}$ timescale, much longer than the ns timescale of most biological autofluorescence [19]. Subsequently, we have seen lanthanides used for high-sensitivity gated detection in immunoassays $[20,21]$ and in DNA hybridization assays [22,23]. The enhanced spectral properties of europium tetracycline described in this paper, suggest that by using metallic particles, even greater sensitivity may potentially be achieved in these sensing platforms.

\section{MATERIALS AND METHODS}

\section{Materials}

All inorganic salts were of analytical purity and were obtained from Sigma-Aldrich (St. Louis, MO). All solutions were prepared in a $10 \mathrm{mM}$ 4-(2-hydroxyethyl)-1piperazineethanesulfonic acid buffer (HEPES, pH 8.0). The luminescent probe europium tetracycline (EuTc, 1:1 ratio), Fig. 1, was prepared from $\mathrm{EuCl}_{3} \cdot 6 \mathrm{H}_{2} \mathrm{O}$ and tetracycline hydrochloride (Sigma-Aldrich).

\section{Preparation of Silver Island Films}

SiFs were formed on aminopropyltriethoxysilane (APS) coated microscope slides (used as received from Sigma-Aldrich) according to previous procedures [1-3].

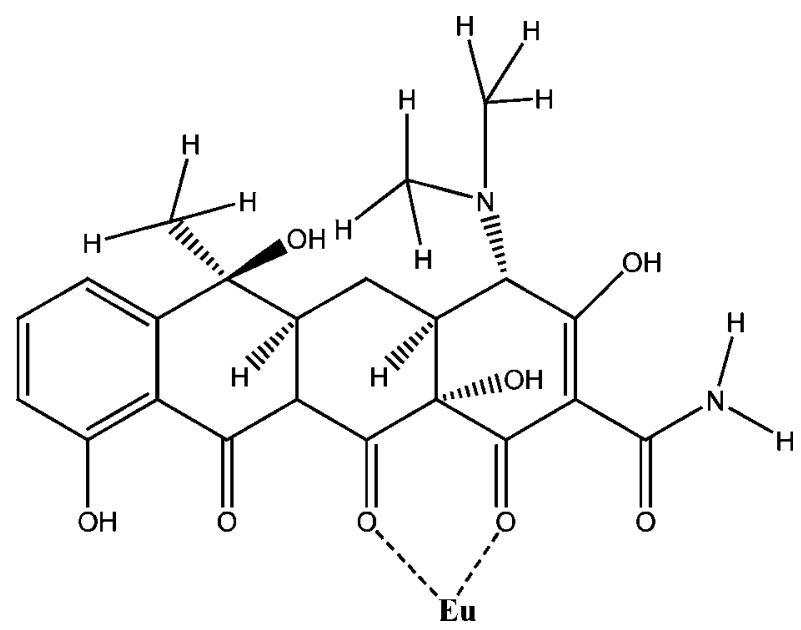

Fig. 1. Molecular structure of tetracycline (Tc), and its coordination with europium (III).
This procedure consists of reducing silver nitrate with D-glucose under controlled conditions and results in a partial coating of the slide with silver islands. The diameters of the islands are typically $100-500 \mathrm{~nm}$ across and some $60 \mathrm{~nm}$ high [1-3]. These nanoparticles display a typical surface plasmon resonance characteristic of subwavelength size silver [14], Fig. 2, with a plasmon absorption band centered near $430 \mathrm{~nm}$. The slides were only half coated with silver, the other half of the substrate providing a reference (the control sample) so as to rational the enhancement due to the SiFs, Fig. 3.

\section{EuTc-PVA Sample Preparation}

The immobilization of EuTc on the surface of the SiFs was achieved by the spin-coating of different mixtures of the EuTc stock solution and $10 \%$ PVA (w/w) aqueous solution on SiFs using a spincoater (Model P6700, from Speedline Technologies, Elgin, IL), at a typical speed of $3000 \mathrm{rpm}$ for 120 seconds. This protocol typically produced films $30-\mathrm{nm}$ thick. In this paper, we have been able to observe the photophysical properties of EuTc spincoated on SiFs as a function of PVA concentration, EuTc concentration in the films, as well as a function of the optical density of the SiFs on the surface.

\section{Methods}

Fluorescence measurements were performed on a Cary Eclipse fluorescence spectrophotometer (Varian, Palo Alto, CA) using the configuration shown in Fig. 4. Absorption measurements were performed using a Hewlett-Packard spectrophotometer (Model 8453, Palo Alto, CA).

Time-resolved intensity decays were recorded using a pulsed xenon arc lamp, pulse width $\approx 2 \mu \mathrm{s}$ fwhm, $200 \mathrm{~Hz}$, and an MSA 300 PC card (Becker and Hickl Gmbh) using an unamplified MCP-PMT. Vertically polarized excitation at $340 \mathrm{~nm}$ was selected using an interference filter (Edmund Scientific) and a prism polarizer, while emission was collected at the magic angle, $54.7^{\circ}$, using a $520 \mathrm{~nm}$ long-pass filter, Fig. 4-bottom.

\section{Data Analysis}

The luminescence intensity decays were analyzed in terms of the multiexponential model:

$$
I(t)=\sum_{i} \alpha_{i} \exp \left(-t / \tau_{i}\right)
$$

where $\alpha_{\mathrm{i}}$ are the amplitudes and $\tau_{\mathrm{i}}$ the decay times, $\Sigma \alpha_{i}=$ 1.0. The fractional contribution of each component to the 


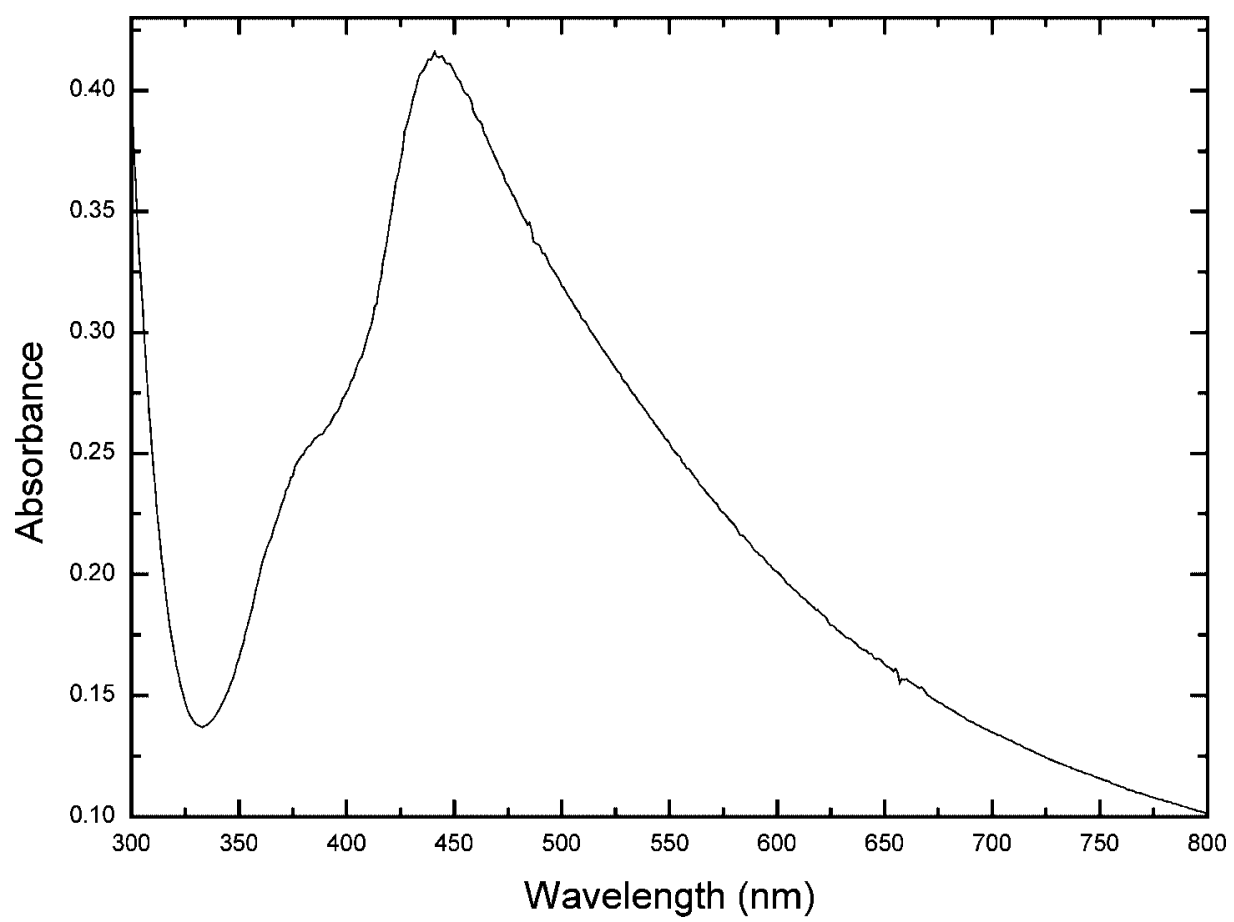

Fig. 2. Typical absorption spectrum of Silver Island Films (SiFs).

steady-state intensity is given by:

$$
f_{i}=\frac{\alpha_{i} \tau_{i}}{\sum_{i} \alpha_{i} \tau_{i}}
$$

The mean lifetime of the excited state is given by:

$$
\bar{\tau}=\sum_{i} f_{i} \tau_{i}
$$

and the amplitude-weighted lifetime is given by:

$$
<\tau>=\sum_{i} \alpha_{i} \tau_{i}
$$

The values of $\alpha_{i}$ and $\tau_{i}$ were determined by nonlinear least squares impulse reconvolution with a goodness-of-fit $\chi^{2} R$ criterion [19].

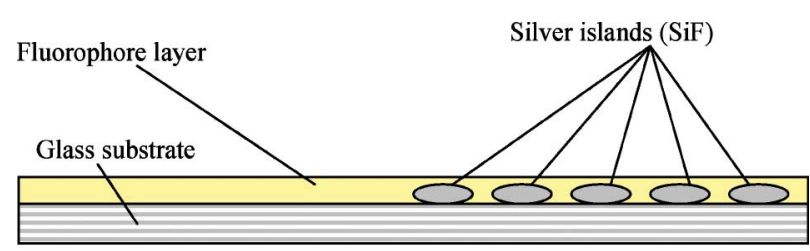

G

S

Fig. 3. Sample Geometry, $\mathrm{S}-\mathrm{SiFs}, \mathrm{G}-$ Glass (Control sample).

\section{RESULTS}

Figure 5-top, shows the emission spectra of EuTC in a $10 \%$ PVA film spin coated on both SiFs and also onto a glass slide, which was used as a control sample. We can clearly see that the overall luminescence is typically greater on the $\mathrm{SiFs}$ as compared to the glass surface. On silver we see two distinct emission bands, the tetracycline emission at $\approx 560 \mathrm{~nm}$ and the $\mathrm{Eu}^{3+} \mathrm{Tc}$ complex (EuTc) at $\approx 615 \mathrm{~nm}$. On the glass surface the emission of the Tc and EuTc bands are less pronounced, Fig. 5-Top. We estimate that there is an approximate ninefold increase in $\mathrm{Eu}^{3+}$ emission on the SiFs, with a 5-7-fold enhancement evident for the TC on SiFs as compared to the glass control sample. To demonstrate that the spectral characteristics for both the Tc and EuTc are the same on both silver and glass, it is informative to normalize both spectra to the EuTc luminescence, Fig. 5-bottom. On the SiFs we typically see a greater EuTc/Tc luminescence ratio, Fig. 5-bottom inserts, increasing from 0.94 to 1.32 on SiFs, a $140 \%$ increase. This suggests that the enhanced Tc emission on the SiFs is facilitating more efficient energy transfer to the $\mathrm{Eu}^{3+}$ in the EuTc complexes. Clearly, the emission of Tc from the PVA films suggests that some Tc is indeed free and uncomplexed. 

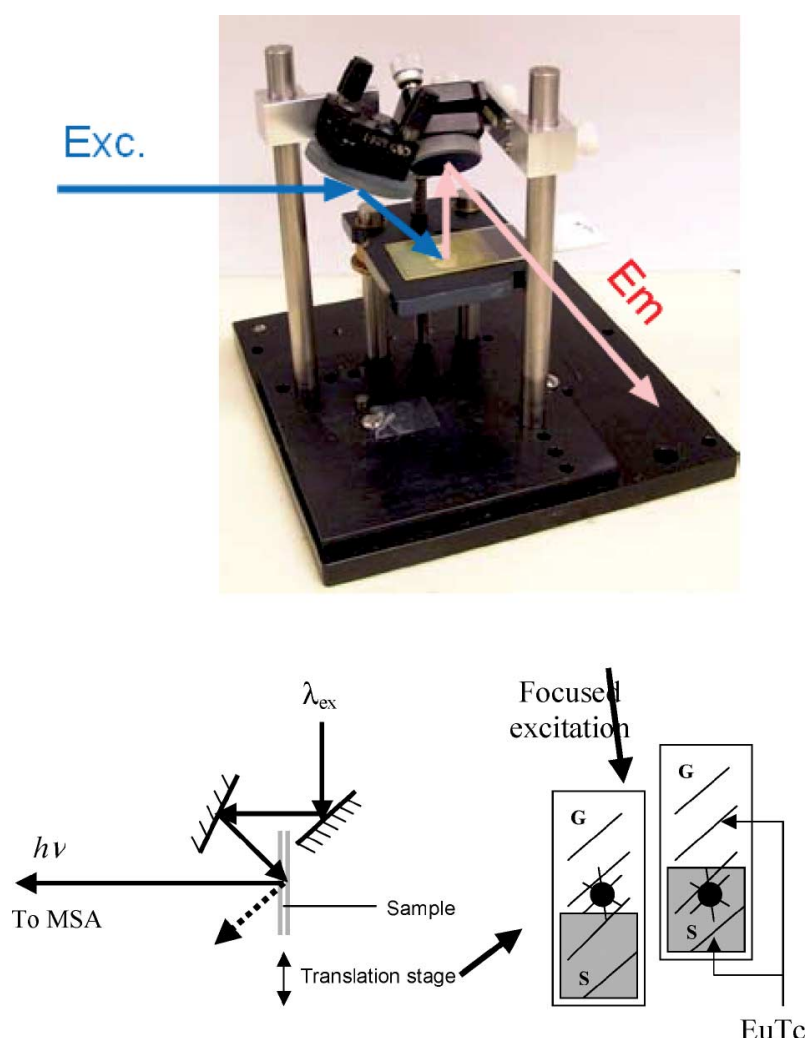

Fig. 4. Geometry used to study steady-state EuTc PVA luminescence (Top), and the geometry used for lifetime analysis (Bottom).

We subsequently prepared Tc doped PVA films spincoated on both SiFs and glass, in an analogous manner to those used above, Fig. 6 . We were able to observe the Tc emission maximum centered around $560 \mathrm{~nm}$, with an approximate sevenfold enhancement seen on $\mathrm{SiFs}$ as compared to the glass substrate. Normalizing the spectra shows the shape of emission spectra to be the same on both $\mathrm{SiFs}$ and glass. It is also worth noting that Tc PVA films were significantly less photostable than the EuTc films on SiFs, which we attribute to both the short lifetime of Tc in the presence of silver nanoparticles, coupled with the reduced Tc lifetime of the EuTc complex due to energy transfer, noting that Fig. 5 suggests two populations of Tc within the films.

It is of interest to calculate the lifetimes of the EuTc on both the SiFs and the control sample as this helps to explain whether a radiative decay rate modification is indeed responsible for our observed enhanced luminescent intensities.

The free space quantum yield of a fluorophore is given by:

$$
Q=\frac{\Gamma}{\Gamma+k_{\mathrm{nr}}}
$$
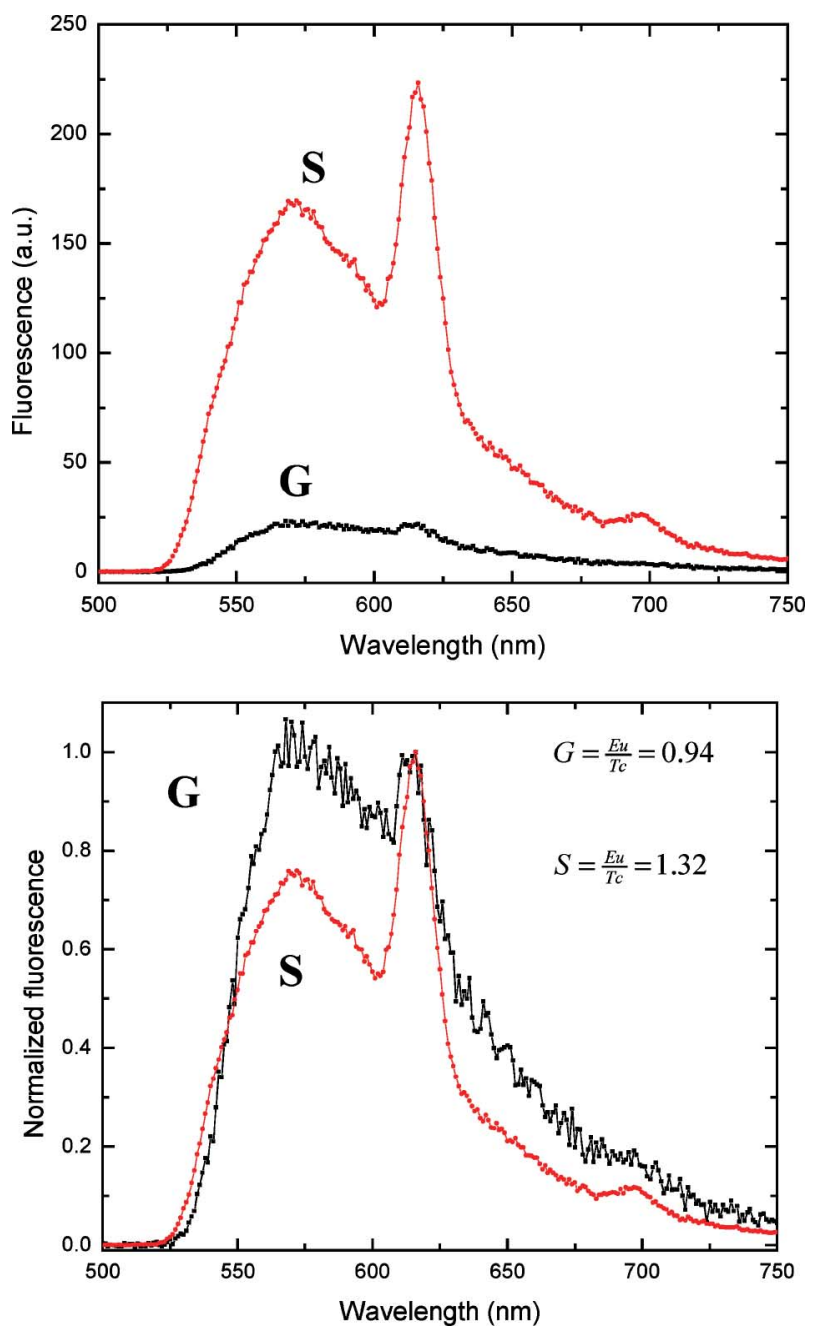

Fig. 5. Luminescence spectra of EuTc on both silver (S) and glass $(\mathrm{G})$ slides (Top), and the spectra normalized to the $\mathrm{Eu}^{3+}$ intensity at $617 \mathrm{~nm}$ (Bottom).

where $\Gamma$ is the radiative decay rate and $K_{\mathrm{nr}}$ are the nonradiative rates. The free space lifetime is given by:

$$
\tau=\frac{1}{\Gamma+k_{\mathrm{nr}}}
$$

If the silver particles modify $\Gamma$ by a factor $n$ and leave $K_{\mathrm{nr}}$ unchanged, then the quantum yield and lifetimes in the presence of metal are given by:

$$
\begin{gathered}
Q_{\mathrm{m}}=\frac{n \Gamma}{n \Gamma+k_{\mathrm{nr}}} \\
\tau_{\mathrm{m}}=\frac{1}{n \Gamma+k_{\mathrm{nr}}}
\end{gathered}
$$

Hence we can see from equations 7 and 8 that an increase in $\Gamma$ both increases the quantum yield while simultaneously reducing the lifetime. This is unusual to many 

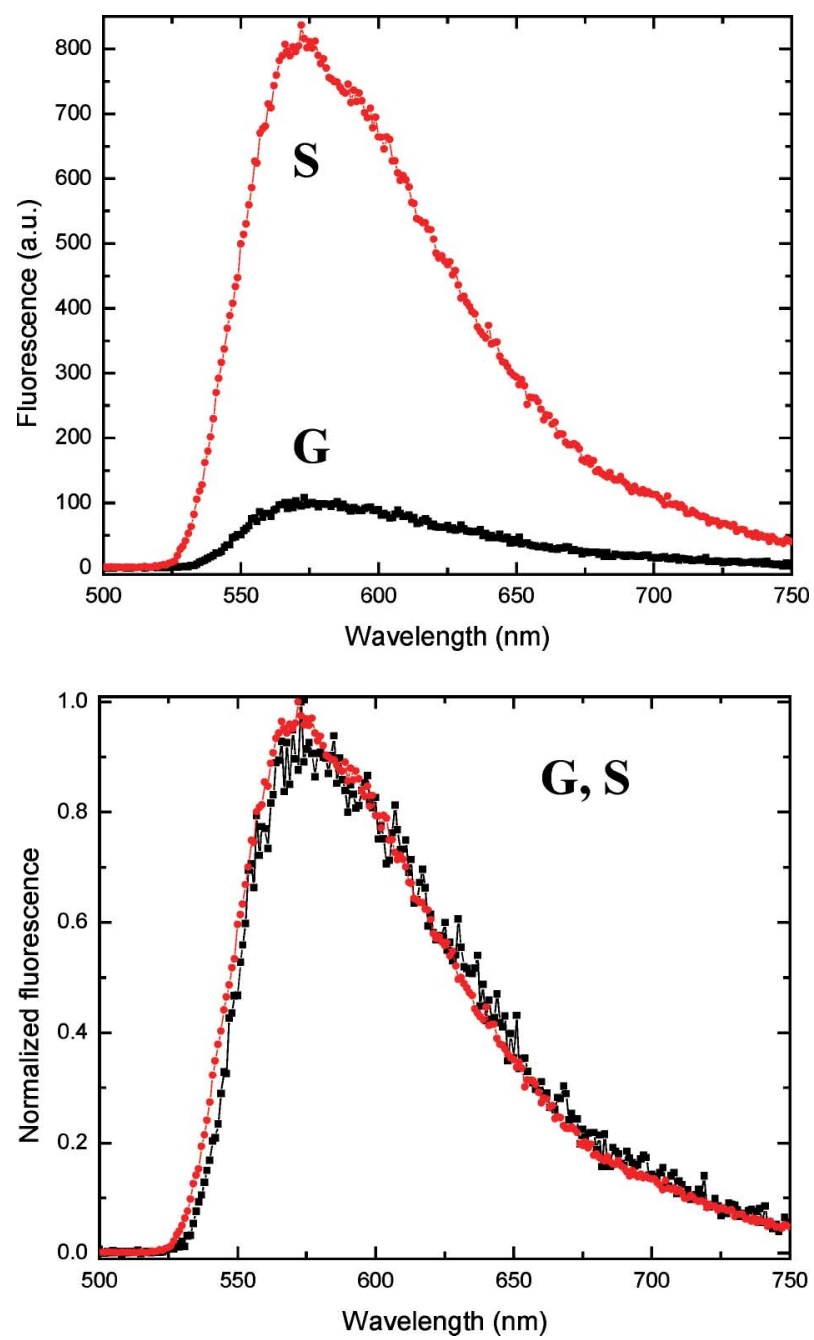

Fig. 6. Luminescence spectra of Tc on both silver (S) and glass (G) slides (Top), and the spectra normalized to the same intensity value (Bottom).

fluorescence spectroscopists as the quantum yield and lifetime usually change in unison $[14,17,19]$.

Figure 7 shows the intensity decays of the EuTc complex in both solution, and PVA spin-coated onto both $\mathrm{SiFs}$ and glass. For clarity the instrumental response function has been omitted. Clearly we can see a significant reduction in lifetime on $\mathrm{SiFs}$ as compared to both solution and immobilized on the glass substrate. Convolution analysis reveals that the mean lifetime is reduced from $12.94 \mu \mathrm{s}$ in solution to $3.45 \mu \mathrm{s}$ on $\mathrm{SiFs}$, an approximate fourfold reduction. Hence an increase in luminescence intensity coupled with a reduction in lifetime, can only be explained by a radiative decay rate modification, cf. equations 7 ad 8 . These trends are similar to those reported in some of our recent reports using nanosecond decay time fluorophores, and both SiFs [1-6], silver colloid films [7] and silver frac-

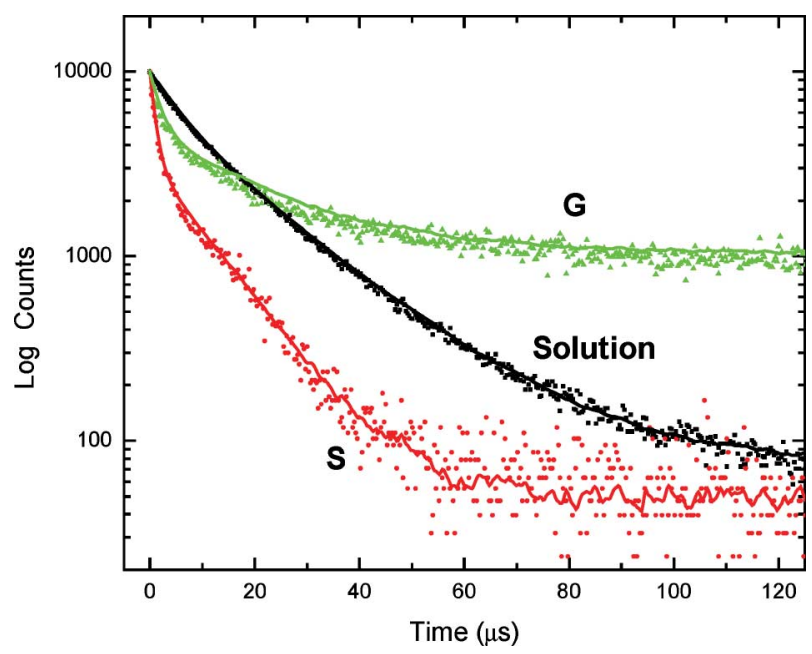

Fig. 7. Luminescence decay profiles for EuTc in solution, and on silver (S) and glass (G) slides. The instrumental response function was removed for clarity.

tals [10-13]. In these past studies the lifetimes of the fluorophores in close proximity to the silver nanostructures were reduced $\approx 100$-fold to less than $100 \mathrm{ps}$, as compared to the $\approx$ fourfold reduction in mean lifetime observed here. While the PVA films used here are thought thin enough to just place the EuTc complex within the active enhancement region of $\sim 20 \mathrm{~nm}$ rationaled by our laboratories to facilitate the MEF phenomenon [1,14,17], it is likely that a much shorter Europium lifetime is present, but not measurable with the time-resolution of the instrument specifically employed here to measure microsecond decay times (see Table 1). Further studies in this regard are underway and will be reported in due course.

By varying the sample preparation conditions to produce slides with a greater density of $\mathrm{SiFs}$, we were able to see somewhat larger luminescent enhancements, Fig. 8. The enhancement, calculated relative to the luminescent intensity observed on glass, was found to increase in a linear fashion with the optical density of the SiFs, measured at the plasmon absorption maxima of $420 \mathrm{~nm}$. This supports recent findings by our laboratories for $\mathrm{SiFs}$ [1] and silver colloid coated films [7] using nanosecond decay time fluorophores.

Table I. Multiexponential Intensity Decay of EuTc in Solution, and Spin Coated on Both Glass Slides and SiFs

\begin{tabular}{lccccccc}
\hline Sample & $\tau_{1}(\mu \mathrm{s})$ & $\alpha_{1}$ & $\tau_{2}(\mu \mathrm{s})$ & $\alpha_{2}$ & $\bar{\tau}$ & $<\tau>$ & $\chi^{2}$ \\
\hline Solution & 6.1 & 0.761 & 19.7 & 0.239 & 12.94 & 9.35 & 1.2 \\
Glass & 1.6 & 0.978 & 24.2 & 0.022 & 7.34 & 7.34 & 1.3 \\
SiFs & 1.1 & 0.970 & 11.1 & 0.030 & 3.45 & 3.48 & 1.2 \\
\hline
\end{tabular}




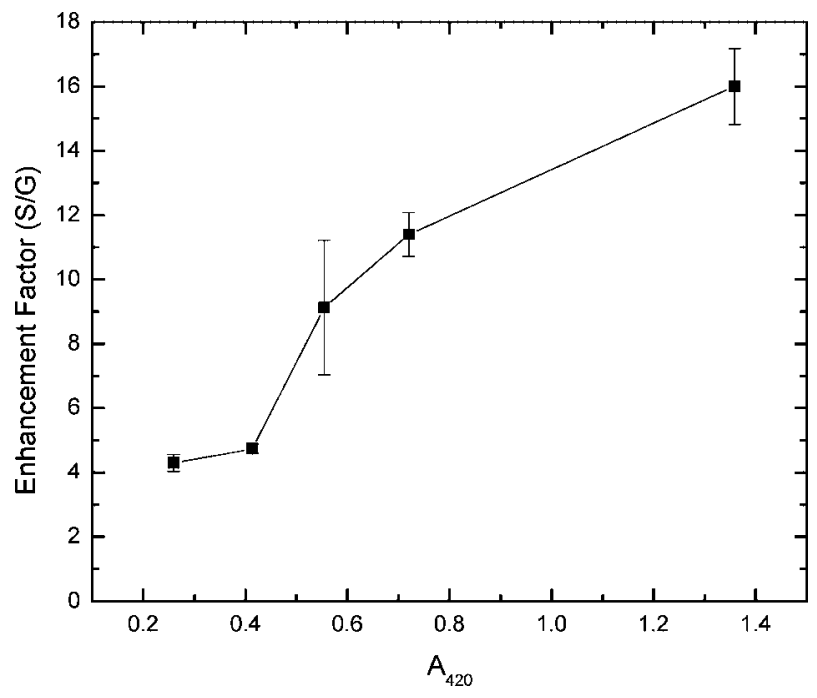

Fig. 8. Enhancement factor (luminescent intensity on silver/glass) for spin-coated PVA EuTc as a function of SiF optical density. Error bars are calculated as the mean of 4 measurements.

We also investigated the effect of the EuTc complex loading into the PVA film with regard to the luminescent enhancement, Fig. 9, as well as the \%PVA used to coat the slides for a fixed $25 \mathrm{mM}$ concentration of complex. We typically found that the luminescence enhancement increased for films comprised of less PVA, which is thought due to the fact that thinner films immobilize a greater frac-

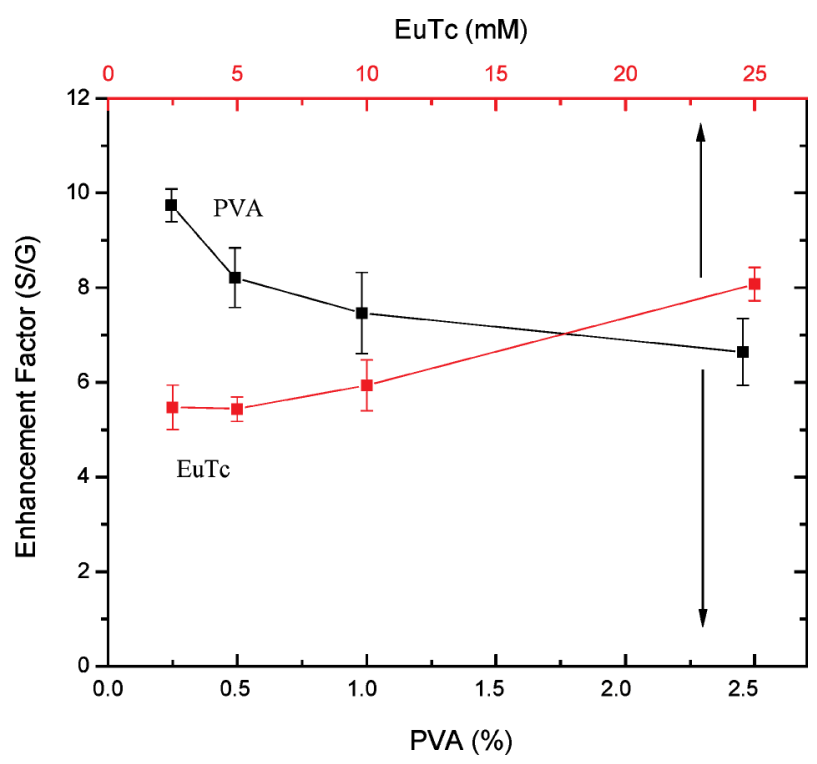

Fig. 9. Enhancement factor (luminescent intensity on silver/glass) for spin-coated PVA EuTc, as a function of EuTc concentration and \%PVA $(\mathrm{w} / \mathrm{w})$. Error bars are calculated as the mean of 4 measurements. tion of the EuTc complex closer to the silver, and therefore just within the $20 \mathrm{~nm}$ enhancement region $[14,17]$.

\section{DISCUSSION}

To place our results in context, it is informative to consider other reports of lanthanides immobilized in close proximity to silver nanostructures [24-27]. While there has been very little work done with lanthanides and silver nanostructures, two reports have found increases in $\mathrm{Eu}^{3+}$ intensity with no change in lifetime [24,25]. An earlier report found a fivefold increase in intensity of a $\mathrm{Eu}^{3+}$ chelate near $\mathrm{SiFs}$, accompanied by a 1000 -fold reduction in lifetime [26]. One other report from our laboratory has recently shown that luminescent enhancements can be observed as complexes diffuse towards the silver nanostructures, during the excited state lifetime of the lanthanides [27], in a solution based sandwich configuration. All these reports which use different lanthanides and chelaters, all serve to both confirm and support our findings that silver nanoparticles can be used to modify the radiative decay rate of lanthanides with favorable spectral characteristics.

In closing it is interesting to speculate on the future potential for lanthanide-silver nanostructures. It is possible that an increased emissive rate could increase the overall detectability. The typical long lifetime of the lanthanides means that they can only emit relatively fewer photons per seconds as compared to ns decay time fluorophores. A substantial decrease in lifetime of a lanthanide-silver structure could therefore allow one to cycle the lanthanide at a much higher rate, increasing detectability. Given the narrow spectral properties of lanthanides, the line spectra due to their transition involving the $4 \mathrm{f}$ orbitals [19], then it may be possible to produce a new unique class of lumophores for applications such as in lanthanide immunoassays [20,21]. Here, the long lived lanthanide luminescence is used to prevail over nanosecond background biological autofluorescence [19], one simply off-gates a detector for several tens of nanoseconds and then observes the lanthanide luminescence. If one could design silver nanostructures that enhance lanthanide luminescence yet drop the lifetime $\approx 100$-fold, still long-enough to off-gate unwanted background fluorescence, then the detectability for immunoassays could be greatly enhanced. Further studies in this regard by our laboratories are underway.

\section{CONCLUSIONS}

By immobilizing EuTc within thin polyvinyl alcohol films in close proximity to $\mathrm{SiFs}$, we can demonstrate 
modifications in the radiative decay rate, evidenced by enhanced luminescence intensities coupled with reduced lifetimes. By varying the sample preparation conditions such as polymer thickness, silver surface density and EuTc concentration, we have been able to modify the enhanced luminescence properties somewhat. Our results support other lanthanide-metal findings.

\section{ACKNOWLEDGMENT}

The authors would like to thank the NIH, National Center for Research Resources, RR-08119, for financial support.

\section{REFERENCES}

1. J. R. Lakowicz, Y. Shen, S. D'Auria, J. Malicka, J. Fang, Z. Gryczynski, and I. Gryczynski (2002). Radiative decay engineering 2. Effects of silver island films on fluorescence intensity, lifetimes, and resonance energy transfer. Anal. Biochem. 301, 261277.

2. J. Malicka, I. Gryczynski, C. D. Geddes, and J. R. Lakowicz (2003). Metal-enhanced emission from Indocyanine Green: A new approach to in vivo imaging. J. Biomed. Opt. 8(3), 472-478.

3. V. J. Pugh, H. Szmacinski, W. E. Moore, C. D. Geddes, and J. R. Lakowicz (2003). Submicrometer spatial resolution of metal-enhanced fluorescence. Appl. Spectrosc. 57(12), 1592-1598.

4. J. R. Lakowicz, J. Malicka, Z. Gryczynski, J. Huang, C. D. Geddes, and I. Gryczynski (2003). Increased sensitivity of fluorescence detection. Pharmagenomics 3(3), 38-46.

5. J. R. Lakowicz, I. Gryczynski, J. Malicka, Z. Gryczynski, and C. D. Geddes (2002). Enhanced and localised multi-photon excited fluorescence near metallic silver islands: Metallic islands can increase probe photostability. J. Fluorescence 12(3/4), 299-302.

6. I. Gryczynski, J. Malicka, Z. Gryczynski, J. R. Lakowicz, and C. D. Geddes (2002). Dramatic increases in resonance energy transfer have been observed between fluorophores bound to DNA above metallic silver islands: Opportunities for long-range immunoassays and new DNA arrays. J. Fluorescence 12(2), 131-133.

7. C. D. Geddes, H. Cao, I. Gryczynski, Z. Gryczynski, J. Fang, and J. R. Lakowicz (2003). Metal-Enhanced Fluorescence (MEF) due to silver colloids on a planar surface: Potential applications of indocyanine green to in vivo imaging. J. Phys. Chem. A. 107, 3443-3449.

8. K. Aslan, J. R. Lakowicz, H. Szmacinski, and C. D. Geddes (2004). Metal-enhanced fluorescence solution based Platform. J. Fluorescence 14, 677-679.

9. K. Aslan, J. R. Lakowicz, and C. D. Geddes (2004). Deposition of silver nanorods on surfaces for applications in Metal-enhanced fluorescence: Interpretation in terms of radiating plasmons. $J$. Phys. Chem. B: in preparation.

10. C. D. Geddes, A. Parfenov, D. Roll, I. Gryczynski, J. Malicka, and J. R. Lakowicz (2004). Roughened silver electrodes for use in metalenhanced fluorescence, Spectrochemica Acta A. 60, 1977-1983.

11. A. Parfenov, I. Gryczynski, J. Malicka, C. D. Geddes, and J. R. Lakowicz (2003). Enhanced fluorescence from fluorophores on fractal silver surfaces. J. Phys. Chem. B. 107(34), 8829-8833.

12. C. D. Geddes, A. Parfenov, D. Roll, I. Gryczynski, J. Malicka, and J. R. Lakowicz (2003). Silver fractal-like structures for metalenhanced fluorescence: Enhanced fluorescence intensities and increased probe photostabilities. J. Fluorescence 13(3), 267-276.

13. C. D. Geddes, A. Parfenov, I. Gryczynski, J. Malicka, D. Roll, and J. R. Lakowicz (2003). Fractal silver structures for metal-enhanced fluorescence: Applications for ultra-bright surface assays and labon-a-chip based nanotechnologies. J. Fluorescence 13(2), 123-128.

14. J. R. Lakowicz (2001). Radiative decay engineering: Biophysical and biomedical applications. Anal. Biochem. 298, 1-24.

15. J. R. Lakowicz, J. Malicka, I. Gryczynski, Z. Gryczynski, and C. D. Geddes (2003). Radiative decay engineering: The role of photonic mode density in biotechnology. J. Physics D. Appl. Phys. 38, R240-249.

16. C. D. Geddes, I. Gryczynski, J. Malicka, Z. Gryczynski, and J. R. Lakowicz (2003). Metal-Enhanced fluorescence: Potential applications in HTS. Combinatorial Chemistry HTS. 6(2), 109-117.

17. C. D. Geddes and J. R. Lakowicz (2002). Metal-enhanced fluorescence. J. Fluorescence 12(2), 121-129.

18. C. D. Geddes, H. Cao, and J. R. Lakowicz (2003). Enhanced photostability of ICG in close proximity to Gold colloids. Spectrochemica Acta A. 59(11), 2611-2617.

19. J. R. Lakowicz (1999). Principles of Fluorescence Spectroscopy, Kluwer Academic/Plenum Publishers: New York, pp. 86-87.

20. I. Hemmila (1993). Progress in delayed fluorescence immunoassay. In O. S. Wolfbeis (Ed.), Fluorescence Spectroscopy: New Methods and Applications, Springer-Verlag, New York, pp. 233-253.

21. J. B. Lamture and T. G. Wensel (1995). Intensely luminescent immunoreactive conjugates of proteins and dipicolinate-based polymeric Tb(III) chelates. Bioconjugate Chem. 6, 88-92.

22. P. R. Selvin and J. E. Hearst (1994). Luminescence energy transfer using a terbium chelate: Improvements on fluorescence energy transfer. Proc. Natl. Acad. Sci. USA 91, 10024-10028.

23. P. R. Selvin (1995). Fluorescence resonance energy transfer. Meth. Enzymol. 246, 301-334.

24. S. T. Selvan, T. Hayakawa, and M. Nogami (1999). Remarkable influence of silver islands on the enhancement of fluorescence from $\mathrm{Eu}^{3+}$ ion-doped silica gels. J. Phys. Chem. B 103, 7064-7067.

25. T. Hayakawa, S. T. Selvan, and M. Nogami (1999). Field enhancement effect of small Ag particles on the fluorescence from $\mathrm{Eu}^{3+}$-doped $\mathrm{SiO}_{2}$ glass. Appl. Phys. Lett. 74, 1513-1515.

26. D. A. Weitz, S. Garoff, C. D. Hanson, T. J. Gramila, and J. I. Gersten (1982). Fluorescent lifetimes of molecules on silver-island films. Optics Lett. 7, 89-91.

27. J. R. Lakowicz, B. P. Maliwal, J. Malicka, Z. Gryczynski, and I. Gryczynski (2002). Effects of silver island films on the luminescent intensity and decay times of lanthanide chelates. J. Fluorescence 12, 431-437. 